\title{
Nach der Wehrpflicht - zwischen internationaler Verantwortung und Risikotransfer
}

\author{
Christian Wevelsiep*
}

\begin{abstract}
The end of conscription for the German Bundeswehr both opens opportunities and carries risks for the future use of military force under German command. This text particularly addresses, on a fundamental level, the question whether a volunteer army can both serve the interests of German security policy and do justice to international conflict prevention and conflict management. It discusses problems to meet international responsibilities to counter security threats with an all-volunteer army, based on a thorough examination of the nature and extent of these responsibilities. The author concludes that such responsibilities can be met by volunteer forces, if properly designed by criteria of international security.
\end{abstract}

Keywords: Military reform, army of volunteers, security policy, international responsibility, risk transfer Wehrreform, Freiwilligenarmee, Sicherheitspolitik, internationale Verantwortung, Risikotransfer

\section{Einleitung}

$\mathrm{D}$ ie Bundeswehr steht bekanntermaßen vor einer der größten Reformen ihrer Geschichte. Während zurzeit etwa eine Viertelmillion Soldaten ihren Dienst leistet, werden es bald erheblich weniger sein, da die Wehrpflicht als eine der Säulen deutscher Sicherheitspolitik ausgesetzt worden ist. Ungeachtet der Dynamik, die das Thema Wehrreform unter dem Sparzwang der öffentlichen Haushalte gewonnen hat, ist noch offen, wie z.B. ein freiwilliger Dienst aussehen könnte; aber ungeachtet praktischer Detailfragen wirft die Wehrreform eine Reihe von ungelösten Fragen auf, die über kurzfristige politische Umsetzungsprobleme hinausgehen. Diese allgemeinen Fragen sollen im Mittelpunkt der folgenden Überlegungen stehen, insbesondere aber soll die Leitfrage, ob es gute Gründe für die Etablierung einer Freiwilligenarmee gibt, in den Fokus gerückt werden,. Kann eine Freiwilligenarmee sowohl den Interessen deutscher Sicherheitspolitik als auch den Aufgaben internationaler Konfliktverhütung und Krisenreaktion gerecht werden? Dieser Frage soll im folgenden nachgegangen werden, wobei das Ziel vorrangig darin liegt, die Argumente für eine Freiwilligenarmee auch gegen die möglichen Gefahren - etwa einer Entgrenzung des Militärischen zu verteidigen. Die Argumentation geht daher in zwei Richtungen: die Denknotwendigkeit des ungerechten Feindes ist im Horizont des liberalen Denkens darzustellen (2.1) und die Kategorie der Verantwortung in internationaler Dimension ist herauszuarbeiten (2.2). Die Etablierung einer Freiwilligenarmee ist vor diesem Hintergrund eine plausible Option, aber es bedarf einer kritischen Rekonstruktion der damit einhergehenden politischen und militärischen Folgeprobleme, insbesondere der Semantik eines „sauberen Kriegs“ (3.). Das Plädoyer für eine Freiwilligenarmee kann daher nur einer skeptischen Position zugrunde liegen (4.).

* Dr. paed. Dr. phil. Christian Wevelsiep ist Privatdozent für Politische Soziologie an der Universität Flensburg. Dieser Beitrag ist referiert.

\section{Aufgaben der Weltinnenpolitik}

\subsection{Die Herausforderung durch den "ungerechten Feind"}

Demokratien fördern im Allgemeinen die Herausbildung und Verdichtung von Kooperation und Interdependenz und sie erhöhen die allgemeinen Anreize für Gewaltverzicht (allgemein: Brunkhorst/Köhler/Lutz-Bachmann 1999, Brock 2000, 2006). Diese höchst erfreuliche „Tradition“ hat einen normativen Kern im Verhältnis von Republikanismus und Frieden (Höffe 1999), aber sie findet eine vorläufige Grenze in der Identifizierung ungerechter Feinde (Kant 1795).

Kants Vorstellung durch Prozesse „wider Willen“ über eine weltbürgerliche Verfassung zu dauerhaftem Frieden zu gelangen, ist eine zentrale, aber nicht unproblematische Zielsetzung (Küng/Senghaas 2003). Zentral darin ist die Annahme einer prinzipiellen Konfliktträchtigkeit von Modernisierungsprozessen, da sich modernisierende Gesellschaften mit sich selbst in Konflikt geraten würden und die so geforderte Kulturaufgabe immer auch wider die eigene Tradition gerichtet ist.

Zur vollen Entfaltung kam der Gedanke des Weltbürgertums im Zeitalter der Aufklärung in der zweiten Hälfte des 18. Jahrhunderts. In seinem Traktat „Zum ewigen Frieden“ hat Immanuel Kant das Weltbürgerrecht in den Zusammenhang einer Begründung einer Weltfriedensordnung gestellt und dabei die Anerkennung eines Weltbürgerrechts im Sinne einer allgemeinen Hospitalität gefordert (Kant 1795, Held 1996). Dieser Zusammenhang zwischen Weltbürgertum und der Friedensförderung eröffnet mehrere Zugänge. Der Kosmopolitanismus repräsentiert den individuellen Anspruch auf internationale Freizügigkeit; und diese zu verwirklichen verlangt, friedliche Verhältnisse zwischen den Staaten und Gesellschaften herzustellen. Von dieser Position aus ergeben sich freilich bedeutende Schlussfolgerungen für das moderne Völkerrecht: Im Rechtszustand zwischen den Staaten gibt es keine gerechten Kriege mehr. Lediglich legale Kriege sind denkbar, die vom völkerrechtlichen Notwehrfall abgesehen nur als Kriege der internationalen Gemeinschaft denkbar sind. Der Angriffskrieg 
wird zum Verbrechen herabgestuft und dessen Bekämpfung wird zu einem erheblichen Teil Gegenstand von Polizeiaktionen (vgl. Brunkhorst 1996, 2002; 2005, S. 14 ff.). Vereinfacht gesprochen, mündet die bis heute gültige Hoffnung einer Kantianischen Perspektive in der Überzeugung, dass durch den Föderalismus freier Staaten die Welt zusammenwachsen und der Krieg schließlich obsolet würde. Den Frieden herzustellen ist eine moralische Pflicht, der Frieden selbst dagegen ist ein Rechtszustand.

Damit kommt mit Kant aber ein weiterer entscheidender Aspekt ins politische Spiel: Trotz aller begründeten Hinwendung zum Pazifismus ist es möglich, Akteure zu identifizieren, die in einem recht- und friedlosen Zustand ihr vorrangiges Interesse sehen. Erst wenn Macht ohne Recht daherkommt und keine Aussicht zu bestehen scheint, dass die Macht auf den Pfad des Rechts verwiesen wird, ist die grundsätzliche Möglichkeit der Ausnahme gegeben. Die Fälle des „failed state“ und des „ungerechten Feindes“ bilden bei Kant solche Ausnahmen. Kant selbst räumt zunächst im Hinblick auf die Existenz eines zerfallenden Staates ein, dass der Fall des Bürgerkrieges die Ausnahme ist. Denn in diesem Fall verschwindet das alle Parteien bindende Recht, der Staat verliert seine Qualität als Rechtsrahmen und büßt somit seine Chance ein, sich in die (gebotene) Richtung des Rechtsstaats und der Republik hin zu entwickeln (Kant 1795, S. 199 ff., Brock 2000, 2006). Wenn aber dieser Weg versperrt ist, kann der Eingriff von außen die einzige Möglichkeit sein, dass das betroffene Gemeinwesen den Entwicklungspfad der praktischen Vernunft wieder aufnimmt. Die Aktualität dieser Ausnahme muss nicht eigens unter Beweis gestellt werden, sie ist im Hinblick auf die Erosion von Staaten in der Peripherie der Moderne evident, aber mit ihr wird zugleich das überlieferte nationalstaatliche Muster hinterfragt, das ausgehend vom klassischen Völkerrecht und dem Prinzip der souveränen Gleichheit der Staaten nun vor eine gewandelte weltpolitische Konstellation gestellt wird: Kant konnte den Angriffskrieg verpönen und das „ius ad bellum“ in Frage stellen, insoweit das Völkerrecht auf die exklusive Teilnahme von Nationen zugeschnitten war, die als Teilnehmer eines strategischen Spiels sowohl ein gehöriges Maß an faktischer Unabhängigkeit genossen als auch ihr Handeln an eigenen nationalstaatlichen Kategorien und Präferenzen orientierten - die formelle Gleichstellung der Völkerrechtssubjekte wurde durch ein eingespieltes Machtgleichgewicht gedeckt (Habermas 2004, S. 117 ff.). Der vielfach hervorgehobene Wandel vom Staatenrecht zum Recht der Weltbürger (Habermas 2004, S. 122) vollzieht einen profunden Wandel im Selbstverhältnis, denn die den Individuen zugeschriebenen Menschen- und Bürgerrechte sollen fortan durch die internationalen Beziehungen hindurch greifen; die Verstaatlichung der internationalen Beziehungen transformiert letztlich auch das äußere Staatenverhältnis.

\subsection{Zur Kategorie der internationalen Verant- wortung}

Die Idee einer vollständigen Konstitutionalisierung des Völkerrechts in Gestalt einer Weltrepublik wurde mit Kant auf den Weg gebracht. Selbst wenn er zwar theoretisch auf halbem
Wege der Einführung einer schwächeren Konzeption des Völkerbundes stehen blieb (Habermas 2004, S. 123 f., Lutz-Bachmann 1999), bleibt doch die Hoffnung auf die friedliche Natur von Republiken, die pazifizierende Kraft des Freihandels und die kritische Funktion einer Weltöffentlichkeit bestehen (zu letzterem Punkt: Brunkhorst 2002, 2005).

Der entscheidende Wandel, der mit der Gründung und Fortentwicklung des älteren Völkerbundes, der UN-Charta und den diversen völkerrechtlichen Innovationen verknüpft ist, kann hier nicht ansatzweise skizziert werden (Dicke 2002, Brunkhorst 1996; Lutz-Bachmann/Bohman 1996). Aber für die vorliegenden Überlegungen ist festzuhalten, dass der Unterstellung der prinzipiellen Unschuld souveräner Völkerrechtssubjekte der Boden entzogen wurde und dass die explizite Verschränkung einer Politik der Friedenssicherung mit einer Politik der Menschenrechte, die Verknüpfung des Gewaltverbots mit der Androhung von auf Sanktionen gestützter Strafverfolgung und die Universalisierung des von der Weltorganisation gesetzten Rechts die entscheidenden normativen Neuerungen im politischen Horizont der internationalen Gemeinschaft darstellen (Habermas 2004, S. 159). Verfassungsprinzipien, die bislang innerhalb von Nationalstaaten verwirklicht wurden, werden weltweit zur Geltung gebracht; die Tatbestände des Friedensbruchs werden im Sinne einer erweiterten Menschenrechtspolitik ausgelegt; zunehmend wird Entwicklungen des Staatszerfalls und massiver Menschenrechtsverletzungen durch die Praxis gewaltverhütender oder friedenerzwingender Interventionen entgegengearbeitet. Der Interventionsverzicht selbst wird begründungspflichtig.

All dies könnte nun zu der Annahme verleiten, dass die angesprochene neue Rolle einer deutschen Freiwilligenarmee in dieses neue Gefüge internationaler Friedenssicherungsmaßnahmen aufgenommen werden sollte: im Sinne einer Fortführung der eingespielten Praxis gewaltverhütender Maßnahmen, im Sinne der weltweit durchzusetzenden Achtung der Menschenrechte und Grundfreiheiten, nicht zuletzt im Sinne einer legitimen Gewaltanwendung, deren einziger Zweck darin liegen kann, Gewalt zu verhindern oder einzudämmen. Ein mögliches Plädoyer für eine Freiwilligenarmee lässt sich insofern aus der gegebenen Situation internationaler Beziehungen und internationaler Konfliktlagen ableiten, geht man davon aus, dass es Menschenrechtsverletzungen gibt, die mit der oben dargestellten Denknotwendigkeit ungerechter Feinde einhergeht. Ein aktueller Fall ist Libyen, hier soll jedoch allgemein dargestellt werden, dass es internationale Verantwortlichkeiten gibt, die ohne Streitkräfte nicht wahrgenommen werden können.

Der Begriff der Verantwortung ist in internationaler Dimension voraussetzungsvoll: Internationale Verantwortung wird dann erforderlich, wenn in zerrütteten Regionen Stabilität und der Aufbau staatlicher Strukturen begleitet oder erleichtert werden soll, wenn Katastrophenhilfe geleistet wird oder internationale Kriminalität zu verhindern ist. Aber der Breite der Aufgaben steht nun bekanntermaßen die Gefahr einer Überdehnung und Überforderung entgegen, sodass sich die Rolle von Militärbündnissen wiederum verändern wird. Diskutiert wird ja etwa die Rückbesinnung der NATO zur ursprünglichen Quintessenz eines klassisch en Sicherheitsbündnisses (Kamp 2010). 
Aber auch dies ist in einem Geflecht von Bedingungen zu betrachten, das keine einfache lineare Entwicklung widerspiegelt. Die traumatischen Einsatzerfahrungen am Hindukusch mögen auf Dauer auf einen militärischen Rückzug hindeuten und das sicherheitspolitische Pendel auf den klassischen Verteidigungsgedanken zurück schwingen - aber dies dispensiert nicht von der Frage, was die Kategorie der Verantwortung im internationalen Maßstab bedeutet, wenn es um den fundamentalen Anspruch der Fürsorge geht.

Die aktuellen Diskussionen um die Schutzverantwortung für die Zivilbevölkerung souveräner Staaten lassen sich hier durchaus als Ausgangspunkt für eine Auseinandersetzung um die Ausgestaltung einer Weltordnung des 21. Jahrhunderts betrachten und sie stehen keineswegs im reinen Widerspruch zur Problematik einer souveränitätstheoretischen Verkürzung des Friedenskontinuums. Das Kontinuum von Frieden, Menschenund Freiheitsrecht ist vielmehr mit dem Fürsorgegedanken in Anschlag zu bringen, es ist dialektisch zu vermitteln: Die Frage, wie die internationale Gemeinschaft mit wachsenden Zonen systemischer Instabilität und dem Auftreten von Massengräueln umgehen soll, ist zwischen Nord und Süd, etablierten und aufstrebenden Mächten nicht beantwortet. Geopolitische und ökonomische Interessen blockieren weiterführende Debatten um Souveränität und Ermächtigungsnormen; die internationalen Entscheidungsfindungsprozesse stocken gerade in den Fällen, in denen eine schnelle Reaktion erforderlich wäre. Insofern ist eine Auseinandersetzung mit dem Konstrukt der Verantwortung notwendig. Denn es stellt ja eine begründete normative Forderung zur Disposition, für die es auf Weltgesellschaftsebene bislang nur halbherzige Lösungen, nicht selten katastrophales Schweigen und Nichthandeln gab.

\section{Das Problem des Risikotransfer-Militarismus}

Die herausgestellte Verschiebung - vom Ideal souveräner Staatenegalität hin zu weltrepublikanischen Strukturen, vom „nationalen“ Selbstinteresse zur Verantwortung und vom Modell der Wehrpflicht hin zu einer international handlungsfähigen Freiwilligenarmee - sieht sich diversen normativen und politischen Dilemmata gegenüber: Wer Völkermord begeht oder ethnische Säuberungen durchführt, bedroht grundlegende Werte und Interessen der Menschheit. „Dagegen muss etwas unternommen werden, in erster Instanz von dem entsprechenden Staat und in zweiter von der internationalen Gemeinschaft. In beiden Fällen erfolgt die Zuschreibung von Verantwortung infolge einer schwerwiegenden Verletzung grundlegender Menschenrechte“ (Welsh 2010, S. 281). Aber obwohl diese Semantik auf eine enge Bindung an verpflichtende Normen zu verweisen scheint, bleibt sie im Rahmen der jeweiligen Verlautbarungen ohne Erwähnung von Handlungspflichten (Rodin 2006, Warner 2003; eine Befürwortung für ein starkes Konzept von Handlungspflichten findet sich hingegen bei Hinsch/Janssen 2006). Genauer gesagt: die von der ICISS vorgeschlagene Formulierung zur Schutzverantwortung verweist semantisch auf eine prospektive Verantwortungskonstruktion, die zugleich politisch Ermessenssache bleibt. Es wird eine Pflicht zu schützen etabliert, aber keine entsprechende „Haftung“ angedacht, wenn dieser Pflicht nicht nachgekommen wird. Dieser Umstand wird von einer Reihe von Theoretikern als grundlegendes Defizit charakterisiert, das zu enttäuschten Erwartungen, mithin zum Fehlen eines Verfahrens führen würde, in dem die internationale Gemeinschaft verantwortlich „gemacht“ werden könnte (Rodin 2006; Warner 2003, Welsh 2010; Dicke 2002; Delbrück 1981, 1999).

An dieser Stelle ließe sich die Kategorie der Verantwortung direkt in ein Plädoyer für eine effektive Freiwilligenarmee ummünzen (Müller 2000), aber es wird dabei unumgänglich sein, die Probleme des Risikomilitarismus in die Überlegungen aufzunehmen. Neben der Hierarchie der Verantwortung und der virulenten Frage, wer der legitime Träger einer Hilfsverantwortung wäre, tritt die nicht zu unterschätzende Frage, wie sich dieser Träger zum Problem der Gewalt verhält, inwieweit er bereit ist, legitime Gewalt (etwa unter Aufbringung eigener Opfer) anzuwenden, ob und wie er überhaupt Gewalt in das Spektrum seiner politischen Möglichkeiten einordnet. Verschiedene Beschreibungen lassen sich in diesem Zusammenhang unterscheiden, etwa dass der Liberalismus eine verdeckte Gewaltreserve aufweise (Müller 2000, 2006), oder von anderer Seite, dass Frieden und Demokratie eine Gleichursprünglichkeit aufweisen (Brock 2000, 2004, 2006).

Insbesondere aber ist auf die nahe liegende Tendenz zum Risikotransfer-Militarismus zu verweisen (Moskos 1990; Shaw 1991; 2003). Diese Tendenz umschreibt die neue Art der Kriegführung, wie sie im Irak oder im Kosovo deutlich wurde: Die für die Interventionsmacht tendenziell risikominimierende Kriegführung konzentriert sich auf chirurgische Eingriffe, bei denen das Leben der eigenen Truppen geschont wird und Kollateraltötungen zwar minimiert werden, aber nicht gänzlich auszuschließen sind. Risikotransfer-Mentalitäten gehen auf technologische Innovationen zurück, haben aber auch einen gesellschaftlich-politischen Hintergrund.

Dem soziologischen Theorem der „postheroischen Gesellschaft“ gemäß lässt die Kriegs- und Opferbereitschaft moderner demokratischer Gesellschaften in dem Maße nach, wie parallel und verstärkend technologische Innovationen die moderne Kriegführung bestimmen (u.a. Münkler 2002, 2006, Helmig 2011). Dieses Theorem gibt der vorliegenden Problematik von daher eine interessante Stoßrichtung, weil sie einen spezifischen Vorrang der Opfervermeidung unterstellt, den es normativ zu prüfen gilt. Das Bild des Krieges wird in diesem Zusammenhang immer weniger von unmittelbaren Erfahrungen, sondern zunehmend durch mediale Berichterstattungen geprägt, die sich etwa durch eine verschleiernde Rhetorik „chirurgischer Luftschläge“ auszeichnet (im folgenden Helmig 2011, S. 94 ff.). Zahlreiche Neuerungen und Veränderungen im Militärwesen werden an technische Innovationen zurückgebunden, die unter „Effizienzgesichtspunkten“ zwar dem Anspruch nach human, weil zielgerichtet sind, aber zugleich das Menetekel einer entmenschlichten Kriegführung darstellen, denn man setzt auf gesteigerte Präzision, gesteigerte Geschwindigkeit und Wirkung, unterliegt dabei aber möglicherweise der Illusion, mit minimalem Aufwand maximale Wirkungen zu erzielen und dabei Opfer sowohl unter der Zivilbevölkerung als auch in den eigenen Reihen vermeiden zu können. Die vereinfachende Rechnung aus militärtechnologischer Sicht lautet, dass Präzi- 
sion und Opfervermeidung die höheren Anschaffungskosten und den Legitimationsbedarf decken - eine zu simple Rechnung, die sich erst dann abzeichnet, wenn man alle sozialen und empirischen Dimensionen in den Zusammenhang mit aufnimmt. Denn das Bild vom sauberen Krieg eröffnet in der politischen Wirklichkeit offenkundig keinen reinen Mittelweg zwischen den Extremen eines Absolutismus der Rechte und einer radikalen Flexibilität des Utilitarismus (Walzer 2003), da es offensichtlich keinen archimedischen Punkt einer risikolosen Kriegführung gibt - in dem Sinne, dass beide Strategien - die absolute Opferbereitschaft und die absolut risikovermeidende Strategie - in moralischer Hinsicht schwierig sind.

Der Vermutung einer postheroischen Disposition ist insofern unter negativen Vorzeichen Recht zu geben: Ein Krieg wird immer auch an der Heimatfront geführt und der politische Rückhalt für Demokratien dementsprechend mitentscheidend. Gelingt es freilich nicht die eigenen Verluste zu minimieren, schwindet die Bereitschaft, die Kosten und Belastungen des Krieges durchzustehen. Den westlichen und postheroisch geprägten Gesellschaften eine niedrige Toleranzgrenze zuzusprechen, ist insofern ebenso plausibel wie die Annahme des Risikotransfers kritisch zu bewerten wäre. Denn werden die Risiken zunehmend auf lokale Verbündete und in letzter Konsequenz auf Zivilisten abgewälzt, kommt es ferner zu Disproportionen zwischen Verlusten westlicher Militärs und lokaler Zivilbevölkerung und greifen zunehmend entpersonalisierte und automatisierte Kriegführungsmechanismen um sich, zieht dies nicht nur konkrete rechtliche Fragestellungen, sondern fundamentale ethische Probleme nach sich (Helmig 2011, S. 102 f.).

\section{Militär und Demokratie}

Im Horizont des Großphänomens Krieg gibt es nun vermutlich keine reine und klare Position, und dementsprechend kann es das Ziel der folgenden Überlegungen auch nicht sein, der Tendenz des Risikotransfers eine moralisch unanfechtbare Position unbedingter Opferbereitschaft entgegenzuhalten. Das hier verfolgte Plädoyer für eine Freiwilligenarmee umreißt vielmehr eine genuin skeptische Position, die für die fundamentalen ethischen Folgeprobleme eine gewisse Widerspruchsoffenheit verlangt. Einerseits gilt die Rechtsnorm, dass kein Unschuldiger im Rahmen einer militärischen Intervention getötet werden darf, andererseits entzieht sich die Logik militärischer Konflikte einem „Rechtsabsolutismus“ (Merkel 2011, S. 70). Das Argument für eine Freiwilligenarmee erschließt sich daher primär aus der Notwendigkeit der Vermeidung einer risikoaversiven und utilitaristisch „blinden“ Kriegführung, das heißt: die sichtbaren Entwicklungen hin zum vermehrten Einsatz von Computersystemen, lasergelenkten Bomben oder dem steigenden Einsatz von Drohnen sind durch die Bereitstellung eines handlungsfähigen Personals zu flankieren. Der gegenwärtige Fokus auf Präzision und Opfervermeidung und die damit einhergehende Verstärkung von Luftstreitkräften ist nachvollziehbar, aber der Einsatz von Truppenverbänden bleibt unverzichtbar, wenn man davon ausgeht, dass es nicht alleine darum gehen sollte, mit minimalem Aufwand maximale Wirkung zu erzielen, bzw. dass die Gefahr von Kollateralschäden eben nicht nur wegen der damit verbundenen negativen politischen Kosten zu vermeiden ist.

Skeptisch ist eine solche Position, weil sie die Widerspruchsoffenheit militärischen Handelns in der Welt des 21. Jahrhunderts nicht unterschätzt: Die Lehren, die aus der Geschichte bzw. aus den Fällen Bosnien, Kosovo, Ruanda oder Darfur zu ziehen sind, legen eine Handlungsnotwendigkeit und damit eine Handlungskapazität nahe. Gleichwohl dürfen sie nicht zu Selbstbeschreibungen führen, die einem Ermächtigungsrationalismus das Wort reden (mit je unterschiedlichen Akzentuierungen: Walzer 2002, Maccarthy 1999, Ignatieff 2003). Es gibt zwar Fälle, die das Menschheitsgewissen schockieren und Konsequenzen für die internationale Gemeinschaft erzwingen: Dies ist evident; freilich wird nicht selten die Rhetorik einer menschenrechtlichen Erzwingungslogik bemüht, die nicht unproblematisch ist. Hier ist aus stringenter rechtsphilosophischer Sicht die Orientierung an den Kategorien der klassischen Friedensphilosophie hilfreich, insofern sie einer möglichen Drift gewaltbewehrter humanitärer Interventionen einen Riegel vorschiebt (u.a. Lutz-Bachmann 1999, Brunkhorst 1999, Maus 1992, dies. 1999). Damit wird das republikanische Postulat einer weitestgehenden Annäherung an die ideale Identität der Gesetzgebenden und Gesetzesunterworfenen einer überhasteten, etwa rein utilitaristischen Interpretation entzogen. Darüber hinaus ist zu betonen, dass die unmittelbare Berufung auf Menschenrechte sowie die interventionsgerechte Interpretation der UN-Charta die Menschenrechte um jene individualistische Dimension verkürzt, die ihre ursprüngliche Bedeutung ausmachte: Denn die militärische Intervention kommt nicht umhin, trotz ihrer Berufung auf das Menschenrecht zugleich fundamentale Rechte zu verletzen, ohne die Zustimmung der Betroffenen als Träger dieser Rechte einzuholen. Menschenrechte laufen in diesem Sinne Gefahr, von subjektiven Rechtsansprüchen zu objektiven Systemzwecken verkehrt zu werden; der enge Zusammenhang von Menschenrecht, Volkssouveränität und Frieden wird dadurch zunehmend porös. Man muss dieses Prinzip nun in gewissem Sinne auch gegen gut begründete Vorbehalte, gegen dramatische Entwicklungen der Weltgesellschaft und gegen die Evidenz der Spaltung in Zentrum und Peripherie durchhalten, um zu ihrem „wahren“ Kern zu gelangen. Perspektiven, die aus dem Skandalon der ungesetzlichen Unterdrückung eines Volkes einen Anlass zur Einmischung erkennen wollen, setzen zugespitzt formuliert, ein Skandalon an die Stelle eines anderen. Die Stiftung eines Rechts auf Intervention mit militärischen Mitteln unterstellt einen Rechtsbegriff, der das Kontinuum von Menschenrecht und Volkssouveränität unterläuft und aus den Menschenrechten Ermächtigungsnormen macht - eine Kritik, die auf die Präzisierung der Rechtsgrundlagen, weniger auf die Betroffenheit angesichts flächendeckender Menschenrechtsverletzungen heutiger Kriege zielt.

Daraus ergibt sich kein „reines“ Plädoyer für eine Entgrenzung der militärischen Gewalt, aber ein - wenn auch minimaler - humaner Impuls für militärische Investitionen, die nicht zwingend dem etablierten Prinzip des politischen Gewaltverzichts entgegenstehen. Es ist eine mögliche Antwort auf fundamentale Dilemma-Situationen, denen sich die politische Reflexion in internationaler Sicht stellen muss. Das Verhältnis von Moderne 
und Gewalt geht über die einfache Kriegsabneigung oder die ebenso einfache Kriegs- und Gewaltbereitschaft hinaus, aber es ist ebenso unbegründet, von rein postheroischen Mentalitäten zu sprechen, wenn man die Dimension internationaler Einsätze betrachtet, in der militärische Solidarbeiträge faktisch geleistet werden. Ist es möglich, dass sich die engere politische Diskussion über den Sinn einer Transformation der Bundeswehr an vergleichbaren Fragen messen lässt? Können sich Diskurse über sicherheitspolitische Aufgaben aus dem engen Rahmen einer Präferenz des Eigenen und latent hegemonialen Engführungen lösen und die Fragen der Schutzverantwortung integrieren? Die Rationalität zukünftiger Diskurse über die Ausgestaltung der Wehrpflicht hängt von der Beantwortung dieser Frage ab.

\section{Literatur}

Brock, L.: Einmischungsverbot, humanitäre Intervention und wirtschaftliche Interessen. In: Menzel, U.: Vom ewigen Frieden und vom Wohlstand der Nationen. Frankfurt a. M.2000, S. 124158.

Ders.: Verlassene Baustellen - Global Governance im Zeichen des Krieges. In: Fues,T./Hippler, J. (Hg.): Globale Politik. Entwicklung und Frieden in der Weltgesellschaft. Bonn: Dietz 2004, S. 58-90.

Ders.: Kriege der Demokratien. Eine Variante des demokratischen Friedens. In: Geis 2006, S. 203-229.

Brunkhorst, H.: Paradigmenwechsel im Völkerrecht? Lehren aus Bosnien. In: Lutz-Bachmann, M./Bohman, J. (Hg.): Frieden durch Recht. Kants Friedensidee und das Problem einer neuen Weltordnung. Frankfurt a. M. 1996, S. 251-272.

Ders.: Menschenrecht und Souveränität - ein Dilemma? In: Brunkhorst, H./Köhler, W.R./Lutz-Bachmann, M.: (Hg.) Recht auf Menschenrechte. Frankfurt a. M. 1999, S. 157-176.

Ders.: Zwischen Kriegseinsatz und Polizeiaktion. Die Legalisierung des Krieges in der Weltgesellschaft. In: Lutz-Bachmann, N./Bohmann, J. (Hg.): Weltstaat oder Staatenwelt? Frankfurt a. M. 2002, S. 65-75.

Ders.: Das freie Recht zum Kriege. Hegemonialer Großraum oder bürgerschaftlicher Konstitutionalismus? - Zwei Begriffe des Rechts. In: Brunkhorst, H./Costa, S. (Hg.): Jenseits von Zentrum und Peripherie. Zur Verfassung der fragmentierten Weltgesellschaft. München und Mering 2005, S. 5-23.

Delbrück, J.: Menschenrechte im Schnittpunkt zwischen universalem Schutzanspruch und staatlicher Souveränität. In: Schwartländer, J. (Hg.): Menschenrechte und Demokratie. Kehl am Rhein 1981, S. 11-26.

Ders.: Effektivität des UN-Gewaltverbots. In: Die Friedenswarte 4. Jg., 1999, Heft Nr. 1, S. 139 ff.

Dicke, K.: Die Aufgaben der Vereinten Nationen in der Staaten- und Gesellschaftswelt des 21. Jahrhunderts. In: Lutz-Bachmann, M./Bohman, J. (Hg.): Weltstaat oder Staatenwelt? Für und wider die Idee einer Weltrepublik. Frankfurt a. M. 2002, S. 32-46.
Geis, A. (Hg.): Den Krieg überdenken. Kriegsbegriffe und Kriegstheorien in der Kontroverse. Baden Baden 2006.

Gerber, D./Zanetti, V. (Hg.): Kollektive Verantwortung und internationale Beziehungen. Frankfurt a. M. 2010.

Habermas, J.: Der gespaltene Westen. Frankfurt a. M. 2004.

Helmig, J.: Militär im 21. Jahrhundert. Technische Entwicklungen und ihre Auswirkungen auf die Kriegsführung. In: Vorgänge 193, 50. Jg. März 2011, Heft 1. S. 94-107.

Hinsch, W./Janssen, D.: Menschenrechte militärisch schützen. Ein Plädoyer für humanitäre Interventionen. München 2006.

Höffe, O.: Demokratie im Zeitalter der Globalisierung. Frankfurt a. M. 1999.

Ignatieff, M.: Empire lite. Nation Building in Bosnia, Kosovo and Afghanistan. London 2003.

Kamp, K. H.: Nach Afghanistan. In: FAZ vom 20. Mai 2010, S. 7.

Kant, I.: Zum Ewigen Frieden. Zitiert nach Werkausgabe Band 11, hg. v. W. Weischedel, Frankfurt a. M. 1977 (1795).

Küng, H./Senghaas, D. (Hg.): Friedenspolitik. Ethische Grundlagen internationaler Politik. München/Zürich 2003, S. 321371.

Lutz-Bachmann, M.: Weltstaatlichkeit und Menschenrechte nach dem Ende des überlieferten Nationalstaats. In: Brunkhorst, H./Köhler, W./Lutz-Bachmann, M. (Hg.): Recht auf Menschenrechte. Demokratie und internationale Politik. Frankfurt a. M. 1999, S. 199- 216.

Ders.: Weltweiter Frieden durch eine Weltrepublik? Probleme internationaler Friedenssicherung. In: Ders./Bohman, J. (Hg.): Weltstaat oder Staatenwelt? Für und wider die Idee einer Weltrepublik. Frankfurt a. M. 2002, S. 32-46.

Lutz-Bachmann, M./Bohman, J. (Hg.): Weltstaat oder Staatenwelt? Für und wider die Idee einer Weltrepublik. Frankfurt a. M. 2002, S. 32-46.

MacCarthy, T.: Über die Idee eines vernünftigen Völkerrechts. In: Lutz-Bachmann, M./Bohmann, J. . (Hg.): Frieden durch Recht. Frankfurt a. M. 1996, S. 200-230.

Maus, I.: Zur Aufklärung der Demokratietheorie. Rechts- und demokratietheoretische Überlegungen im Anschluss an Kant. Frankfurt a. M. 1992.

Dies.: Volkssouveränität und das Prinzip der Nichtintervention in der Friedensphilosophie Immanuel Kants. In: Brunkhorst, H.: Einmischung erwünscht? Menschenrechte und bewaffnete Intervention. Frankfurt a. M. 1998, S. 88-117.

Dies.: Menschenrechte als Ermächtigungsnormen internationaler Politik oder: der zerstörte Zusammenhang von Menschenrechten und Demokratie. In: Brunkhorst/Köhler/LutzBachmann 1999, S. 276-293.

Ders.: Verfassung oder Vertrag. Zur Verrechtlichung globaler Politik. In: Niesen, P./Herborth, B.: Anarchie der kommunikativen Freiheit. Frankfurt a. M. 2007, S. 350-383. 
Merkel, W.: Demokratie durch Krieg? In: Vorgänge 193, 50. Jg., März 2011, Heft 1, S. 53-79.

Moskos, C. C.: Streitkräfte in einer kriegsfreien Gesellschaft. In: Sicherheit und Frieden, Vol.8. , 1990 (2), S. 110-112.

Müller, H.: Demokratie ohne Armee? Die Forderung nach Auflösung „Stehender Heere“ und die Diskussion um die Umstrukturierung der Bundeswehr, In: Menzel, U.: Vom ewigen Frieden und vom Wohlstand der Nationen. Frankfurt a. M. 2000, S. $102-124$.

Ders.: Kants Schurkenstaat: Der ungerechte Feind und die Selbstermächtigung zum Kriege. In: Geis 2006, S. 229-251.

Münkler, H.: Über den Krieg. Stationen der Kriegsgeschichte im Spiegelt ihrer theoretischen Reflexion. Weilerswist 2002.

Ders.: Der Wandel des Krieges. Von der Symmetrie zur Asymmetrie."Weilerswist 2006.

Rodin, D.: The Responsibility to Protect and the Logic of Rights. Programme for Strategic and International Security Studies. Genf 2006.

Senghaas, D.: Wohin driftet die Welt? Über die Zukunft friedlicher Koexistenz. Frankfurt a. M. 1994.

Shaw, M.: Post-Military society. Militarism, Demilitarization an War at the End of the Twentieht cnetury. Philadelphia 1991.

Ders.: War and Genocide. Organized Killing in Modern Society. Cambridge 2003.

Walzer, M.: Also, ist dieser Krieg gerecht? Eine moralische Erörterung anhand eines aktuellen Beispiels. In: Die Welt vom 29. 3. 2003. S. 1

Warner, D.: The Responsibility to Protect and Irresponsible, Cynical Engagement. In: Millenium. Journal of International Studies 32, 2003, röm.1 S. 109-121.

Welsh, J. M.: The Responsibility to Protect: securitizing the Individual in International Society. In: Goold, B./Lazarus, L. (Hg.): Security and Humann Rights. Oxford 2007, S. 363-383.

Dies.: Die internationale Gemeinschaft und die Verantwortung zum Schutz. In: Gerber/Zanetti 2010, S. 272-295.

Wheeler, N. J.: Saving Strangers. Humanitarian Intervention in International Society. Oxford 2000.

\section{Entwicklungshilfe}

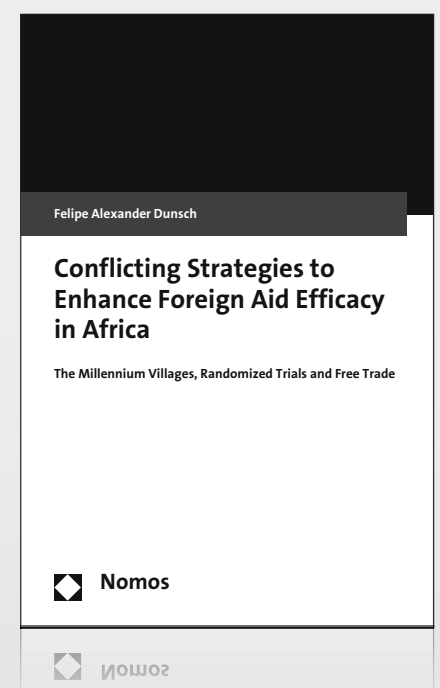

\section{Conflicting Strategies to Enhance Foreign Aid Efficacy in Africa}

The Millennium Villages, Randomized Trials and Free Trade

Von Felipe Alexander Dunsch

2012, 160 S., brosch., 26,- €, ISBN 978-3-8329-7151-9

Warum ist Entwicklungshilfe in der Vergangenheit gescheitert, und welche Lösungen gibt es für die Zukunft? Der Autor erläutert die aktuelle Debatte aus drei verschiedenen Blickwinkeln und bewertet den Nutzen der darauf aufbauenden Trends: Die Millennium Villages der UN, die Randomized Controlled Trials sowie den Mythos des Freihandels als Lösung für Entwicklung.

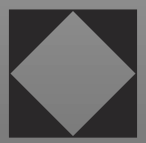

